\title{
The Influence of Pipe Types on The Thermal Performance of Flat-plat Closed Loop Pulsating Heat Pipe
}

\author{
Qingping $\mathrm{Wu}^{1, *}$, Rongji Xu ${ }^{2}$, Ruixiang Wang ${ }^{2}$, Yanzhong $\mathrm{Li}^{1}$ \\ 1. School of Energy and Power Engineering, Xi'an Jiaotong University, Xi'an, China, 710049 \\ 2. School of Environment and Energy Engineering, Beijing University of Civil Engineering and Architecture, Beijing, China, 100044
}

\begin{abstract}
Flat-plat pulsating heat pipes (FCLPHPs) have a great potentiality in electronic cooling field and space application. In this investigation, three FCLPHPs (L1, L2, and L3) were built to study the influence of cross section shapes on the heat transfer performance of FCLPHP. One (L1) of them has asymmetric pipe, the others (L2, L3) have symmetric pipes. The results indicate that the FCLPHP L1 has the best heat transfer performance. Compared with the FCLPHPs L2 and L3, the start-up time reduces by $64 \%$ and the thermal resistance reduces by at most half respectively.
\end{abstract}

\section{Introduction}

In 1990, pulsating heat pipe (PHP) was firstly proposed by Akachi ${ }^{[1,2]}$. It has a broad research prospects and a great practical potential in the field of electronic cooling and space applications.

The researchers have carried out research on the factors affecting the heat transfer performance of pulsating heat pipe, which include the flow pattern of working fluid ${ }^{[3]}$, the thermal physical property ${ }^{[4]}$, the geometric size ${ }^{[5]}$, the filling ratio ${ }^{[6,7]}$, the inclination angle ${ }^{[8]}$ and the heat load ${ }^{[9]}$, etc.

Compared with the symmetric pipe applied in FCLPHP, asymmetric pipe has the advantage of heat transfer performance. As there are few studies considering the performance of FCLPHP with asymmetric pipe, investigation on it is still needed. The experiment hereby was carried to characterize the effect of the pipe types on the heat transfer performance of FCLPHP.

\section{Experiment setup}

The FCLPHPs used in the experiment are schematically shown in Fig. 1. Each FCLPHP was fabricated from an aluminum flat plate. Eight channels were machined into the plate and joined end to end to constitute a whole flow channel. A number of narrow slits were machined into the bottom surface of the flat plate to accommodate Type- $T$ thermocouples.

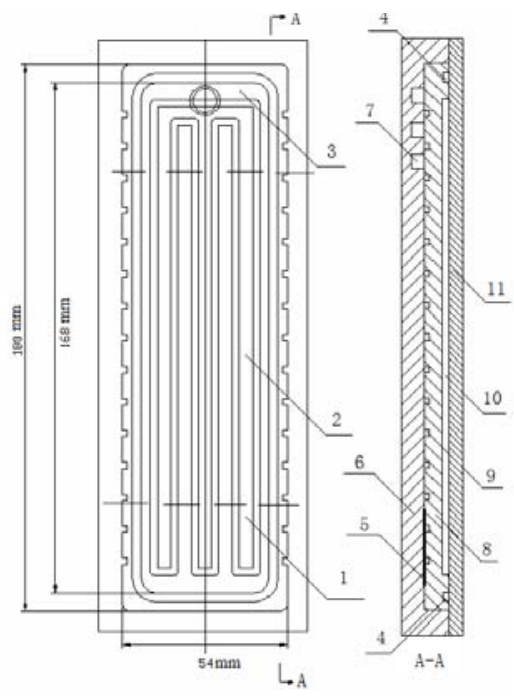

1 Evaporating section, 2 Middle section, 3 Condensing section, 4 Seal groove, 5 Resistor, 6 Adiabatic base,

7 Cooling water channels, 8 Aluminum flat plate, 9 Slit, 10 Flow channel, 11 Plexiglas cover

Fig. 1 Schematic of FCLPHP

The whole FCLPHP was approximately divided into three sections: an evaporating section, a middle section and a condensing section. The aluminum flat plate was placed in an adiabatic plastic base. A cooling water channel was used to cool the working fluid on one side of the base, while a resistor was used to heat the working fluid on the other side of the base. The aluminum flat plate was covered with Plexiglas to allow the observation of the flow of the working fluid. 


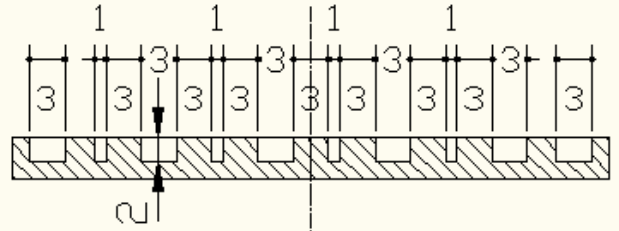

(a) Interval rectangle pipe L1 (asymmetric)

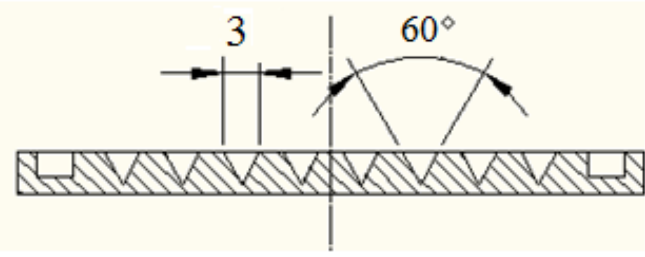

(b) Triangular pipe L2 (symmetric)

5 525,5255

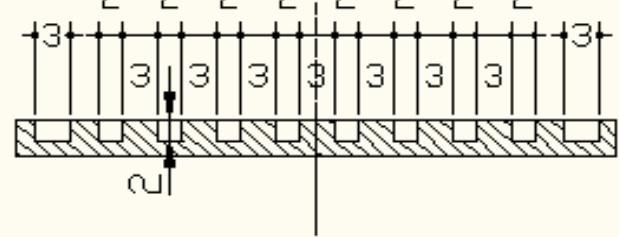

(c) Rectangle pipe L3 (symmetric)

Fig. 2 Cross section of aluminum plate

The capillary channels (as shown in Fig.2) with the different geometrical dimensions and cross-section shapes were experimentally studied. Through the experiments, the heat transfer performances of the FCLPHPs with the interval rectangle cross-section (Fig.2 (a), L1), triangular cross-section (Fig.2 (b), L2) and rectangle cross-section (Fig.2 (c), L3) were compared.

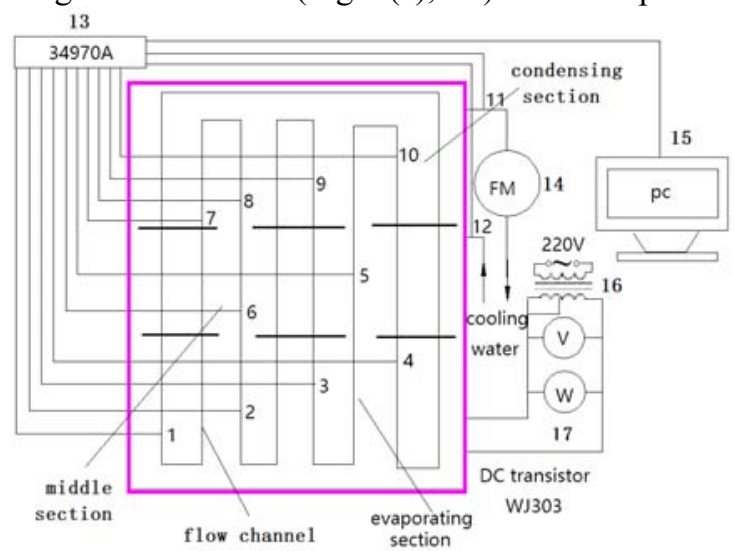

1-12 Type- $T$ thermocouples 13 Data acquisition device,

14 Flow-meter, 15 PC, 16 DC-transistor, 17 Power meter

Fig. 3 Schematic of experimental set-up

Fig. 3 shows the schematic of experimental set-up, which was also used in our previous studies ${ }^{[10]}$. The power was supplied through a DC-transistor WYJ303, the electrical power input was measured by a power meter D26-W with an accuracy of $\pm 0.5 \%$. The flow rate of the cooling water was controlled through adjusting the opening of a valve and was measured by means of a LZB-2 rotary flow-meter with an accuracy of $\pm 2.5 \%$. The temperature measurement system consisted of 10 Type-T thermocouples, a data acquisition instrument Agilent 34970A, and a Personal Computer (PC). The locations of the thermocouples are schematically illustrated in Fig 3. The thermocouples were calibrated with a standard thermometer with a measurement accuracy of $\pm 0.5 \mathrm{~K}$.

The combined standard uncertainty of temperature,

$T$, and heat load, $Q$, are $u_{c}(T)=0.7 \mathrm{~K}$ and $u_{c}(Q)=0.3 \mathrm{~W}$, as shown in Reference [10].

\section{Experimental results and discussion}

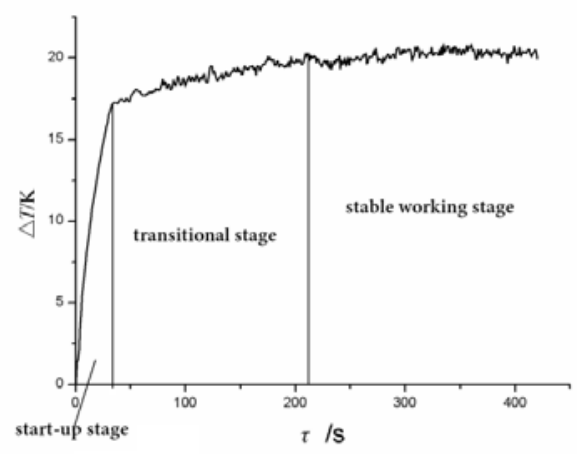

(working fluid: ethanol; heat load: 10.1W; filling ratio: $50 \%$; tilt angle: $70^{\circ}$ )

Fig. 4 Change of temperature

difference between the evaporator and condenser

According to the changes of temperature difference $\Delta T$ between evaporating section and condensing section with time $\tau$, the working process of FCLPHP could be divided into three stages: the start-up stage, the transitional stage and the stable working stage.

In the start-up stage, liquid plugs and vapor slugs oscillated in channels. However, one-direction flow was not observed. The change of the $\Delta T$ vs. time $\tau(\Delta T-\tau)$ in this stage is a smooth curve, as shown in Fig.4.

In this paper, start-up time is defined as a period from the time that a FCLPHP starts to be heated to the time that working fluid starts to boil and $\Delta T$ - $\tau$ curve starts to show fluctuations (see Fig. 4). Start-up time is an important index to evaluate the heat transfer performance in start-up stage. The shorter the start-up time is, the lower the temperature of the evaporating section should be, thus more favorable to the safe and reliable operation of the cooled device.

In the stable working stage, liquid slugs in the evaporating section boil violently, and the working fluid forms circulating flow. $\Delta T$ stops increasing but fluctuates around a mean value. Latent heat exchange becomes important due to the strong boiling and condensing of working fluid.

The thermal resistance $R_{\mathrm{PHP}}$ of FCLPHP is a main index to evaluate the thermal performance of FCLPHP in stable working stage. It is defined as the ratio of temperature difference $\left(T_{\mathrm{e}}-T_{\mathrm{c}}\right)$ between evaporating section and condensing section to heat load $Q$.

$$
R_{P H P}=\left(T_{e}-T_{c}\right) / Q
$$

where $T \mathrm{e}$ is evaporating temperature, $\mathrm{Tc}$ is condensing temperature. 


\subsection{The influence of pipe type on the thermal performance of FCLPHP in start-up stage}

Three FCLPHPs with the different types of pipe (L1, L2 and L3) were tested in the experiment. Fig.5 shows the start-up time of FCLPHPs. In the experiment, working fluid was ethanol, the heat load was $10.2 \mathrm{w}$, the tilt angle was $50^{\circ}$, and the filling rate was $70 \%$.

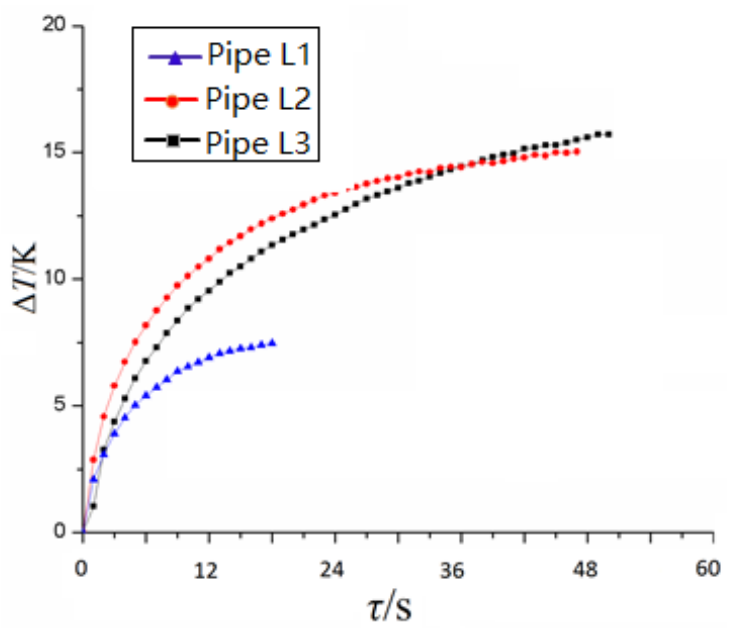

Fig. 5 Influence of pipe type on start-up time of

\section{FCLPHPs}

It can be seen from the Fig.5 that the start-up time of the FCLPHP with interval rectangle cross-section (L1) is the shortest. Compared with that of the FCLPHP with rectangular cross-section (L3), the start-up time is reduced from 50 seconds to 18 seconds, a reduction of $64 \%$.

Adopting asymmetric pipe rather than symmetric pipe, the FCLPHP L1 has more significant flow resistance difference between the thick and thin channels, and greater difference of the intensity of the liquid plugs and vapor slugs vibration between the adjacent channels in the start-up stage, which make the working fluid easier to enter another channel from one channel through the U-shaped elbow in the condensation section, shortening the start-up time.

\subsection{The influence of pipe type on the thermal performance of FCLPHP in stable working stage}

The heat transfer performances of the FCLPHPs with the interval rectangular (L1), triangular (L2) and rectangular cross-section (L3) were compared. In the experiment, the filling rate was $70 \%$, the inclination angles were $50^{\circ}$ and $90^{\circ}$ respectively, and the working fluid was ethanol. The experimental results are shown in Figure 6.

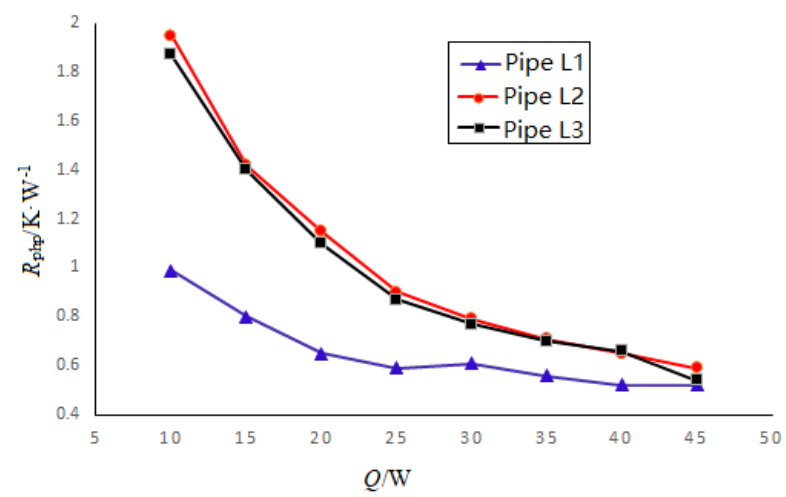

(a) ethanol; filling ratio: $70 \%$; tilt angle: $50^{\circ}$

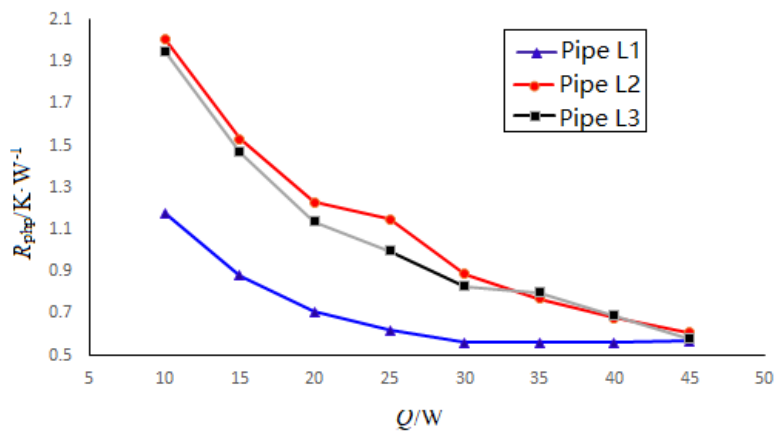

(b) ethanol; filling ratio: $70 \%$; tilt angle: $90^{\circ}$

Fig.6 Influence of pipe type on the thermal performance of FCLPHP in stable working stage

It can be seen that the heat transfer performance of FCLPHP L1 is the best, especially under low load. Compared with the FCLPHPs L2 and L3, the thermal resistance of the $\mathrm{L} 1$ is only about half of that of the other two FCLPHPs when the heat load is $10 \mathrm{~W}$.

When the cross-section shape of FCLPHP is interval rectangle, the different sizes of adjacent channels make the flow resistance difference in the two channels greater, which is conducive to the circulation flow of working fluid and improves the flow velocity of working medium. When the heat load is low, the pressure difference between evaporation section and condensation section is small, the advantage of pulsating heat pipe with asymmetric cross section is more prominent.

\section{Conclusions}

An experimental investigation was conducted to study the influence of pipe types on the heat transfer performance of FCLPHP, the conclusions can be drawn as follows:

(1) According to the changes of temperature difference $\Delta T$ between evaporating section and condensing section with time $\tau$, the working process of FCLPHP could be divided into three stages: the start-up stage, the transitional stage and the stable working stage.

(2) In the start-up stage, the start-up time of the FCLPHP L1 is significantly less than that of the FCLPHPs L2 and L3 because the difference of the liquid plugs and vapor slugs vibration between the adjacent channels of FCLPHP L1 is larger than that of the FCLPHPs L2 and L3. 
(3) In the stable working stage, the experimental results indicate that the thermal resistance of FCLPHP L1 reduces about half compared with the FCLPHPs L2 and L3, because the working fluid in the asymmetric pipe can flow more easily, thus the heat transfer performance of FCLPHP is enhanced.

\section{References}

1. H. Akachi, U.S. Patent No. 4921041 (1990).

2. H. Akachi, F. Polasek, P. Stulc, Pulsating heat pipes, in: Proceedings of the 5th International Heat Pipe Symposium, Melbourne, Australia, pp. 208-217 (1996).

3. Ayel V, Araneo L, Marzorati P, et al. Visualization of Flow Patterns in Closed Loop Flat Plate Pulsating Heat Pipe Acting as Hybrid Thermosyphons under Various Gravity Levels[J]. Heat Transfer Engineering, 2018:00-00.

4. Han H, Cui X, Zhu Y, et al. A comparative study of the behavior of working fluids and their properties on the performance of pulsating heat pipes (PHP) [J]. International Journal of Thermal Sciences, 82(1):138-147 (2014).

5. Yang H, Khandekar S, Groll M. Operational limit of closed loop pulsating heat pipes [J]. Applied Thermal Engineering, 28 (1): 49-59 2008 (2008).

6. Himel Barua, Mohammad Ali, et al. Effect of filling ratio on heat transfer characteristics and performance of a closed loop pulsating heat pipe[J]. Procedia Engineering 56:88 - 95 (2013).

7. $\mathrm{Li} \mathrm{M}, \mathrm{Li} \mathrm{L}, \mathrm{Xu} \mathrm{D}$. Effect of filling ratio and orientation on the performance of a multiple turns helium pulsating heat pipe[J]. Cryogenics, 2019, 100:62-68.

8. Srikrishna P, Siddharth N, Reddy S U M, et al. Experimental investigation of flat plate closed loop pulsating heat pipe[J]. Heat \& Mass Transfer, 55:2637-2649 (2019).

9. Shang F M, Fan S L, et al. An experimental investigation on heat transfer performance of pulsating heat pipe [J]. Journal of Mechanical Science and Technology. 34 (1) : 425-433. (2020)

10. Wu Q P, Xu R J, Wang R X, Li Y Z, Effect of C60 Nanofluid on the Thermal Performance of a Flat-Plate Pulsating Heat Pipe[J]. International Journal of Heat and Mass Transfer, 100:892-898 (2016). 